\title{
- Considérations générales sur la politique de l'eau au XXIe siècle
}

- General considerations on the water policy in the $21^{\circ}$ century -

\author{
par Pierrette Larivaille \\ Adjoint au délégué régional Ile-de-France \\ Electricité de France - Gaz de France
}

\section{La consommation de matières premières, la destruction de la biosphère et surtout l'accroissement de la demande en eau sous la poussée démographique, autant de problèmes qui vont influer sur l'économie mondiale dans les cinquante prochaines années.}

Le cadre au sein duquel les politiques que nous élaborons aujourd hui seront amenées à jouer un rôle peut désormais être relativement bien cerné, sinon dans le détail, du moins par les inévitables bouleversements dont il sera le théâtre.

En effet, les défis des cinquante prochaines années sont inscrits dans les dysfonctionnements qui, depuis plus de deux décennies, affectent de plus en plus gravement non seulement les pays industrialisés mais encore les pays du Tiers Monde.

C'est ainsi que nous assistons à une dissociation toujours plus marquée, d'une part entre la croissance économique et la consommation de matières premières, et d'autre part entre la sphère fiduciaire et l'économie réelle. Ce double phénomène s'accompagne d'une montée des interdépendances à l'échelle mondiale, la richesse ne dépendant plus comme par le passé de la possession du sol et de ses ressources, mais de la maîtrise des savoir-faire technologiques.

Un autre problème se pose à la communauté internationale : la destruction de la biosphère.

Or, au cœur de cette problématique économique et politique, au carrefour du bien-être des hommes et de la survie de la biosphère, un élément fondamental, parmi d'autres certes, mais fondamental tout de même, pose des problèmes qui ne peuvent être élucidés : l'EAU, l'eau qui, pour le dire comme Bachelard, est " l'œil de la Terre " par lequel se voient les dysfonctionnements qu'engendre notre technocosme.

Qu'en est-il alors du cadre général de l'avenir de l'eau ? Il semble qu'il devrait se caractériser par deux tendances, respectivement d'ordre " physique " et " psychologique ", à savoir : une augmentation des besoins et une évolution des mentalités.

Sous l'effet de la poussée démographique mondiale, la demande en eau va inéluctablement croître dans les prochaines décennies.

En effet, même dans le cadre de la prévision non catastrophique de l'ONU, d'une stabilisation de la population mondiale à 10,2 milliards d'habitants à la fin du siècle prochain, le chiffre de 9,5 milliards sera atteint dès 2050 . Or, si l'on se souvient que la population mondiale, s'élevait à 2,5 milliards en 1950 et à 5 milliards en 1990 , c'est donc à un doublement en l'espace d'un siècle (1950-2050) auquel aura à faire face l'humanité.

Based on our experience and the numerous problems we face, the water policies must take into account the qualitative and quantitative aspects of this natural ressource. The qualitative aspect concerns not only the Third World countries with health problems but also the Western countries where industry endanger water quality. The quantitative aspect is also very important because of the natural disparities of distribution and of the phenomena such as deforestation and the greenhouse effect. Decisions must be rapidly taken but nothing will be done if we do not change our relationship with the environment. 
Fait aggravant, outre la menace que font peser sur une partie de notre approvisionnement en eau les évolutions potentielles du climat terrestre, l'expérience de ce siècle montre que la consommation d'eau augmente beaucoup plus vite que la taille de la population puisqu'un quadruplement de celle-ci a entraîné un décuplement de celle-là. Sans parler des disparités naturelles en termes de ressources, que ce soit à l'intérieur d'un même espace national ou entre les pays ; sans compter aussi qu'un certain nombre de pays reçoivent une partie de leur eau de l'extérieur par des fleuves frontaliers.

On imagine alors facilement les tensions qui ne vont pas manquer de résulter de cet accroissement de la demande qui portera -est-il besoin de le préciser ?- non seulement sur la quantité mais également, pour les pays du Tiers Monde, sur la qualité.

Heureusement, ce nouveau défi s'inscrit dans le cadre d'un changement d'attitude par rapport aux questions d'environnement, changement d'attitude intervenu dans l'ensemble des pays industrialisés et qui semble désormais profondément enraciné : le pro-

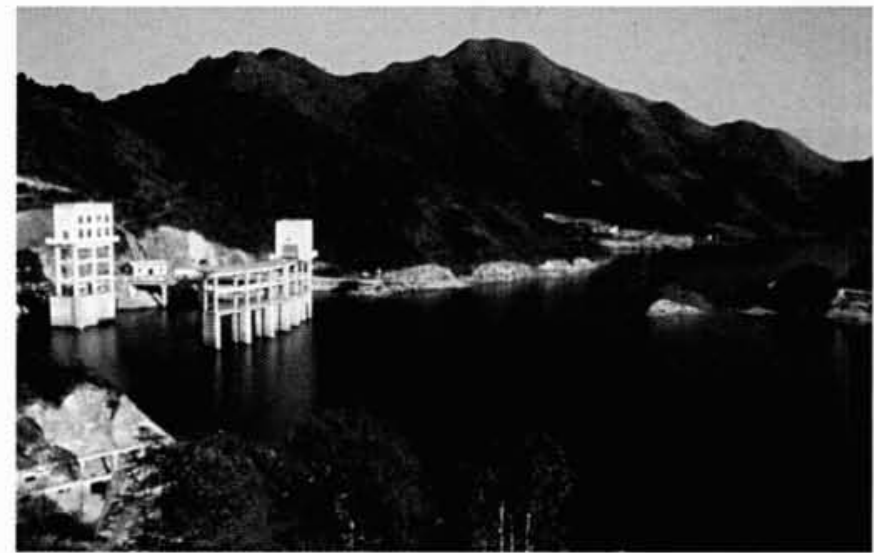

Barrage et retenue d'eau en Chine.

(photothèque EDF - photo Marc Morceau) l'eau des rivières et des lacs au premier rang des préoccupations des Français.

Tous ces événements, tous ces commentaires ont peutêtre finalement joué un rôle semblable à celui de la découverte au $\mathrm{XIX}^{\mathrm{e}}$ siècle du lien entre insalubrité et maladies, découverte qui fut à l'origine de la construction du tout-à-l'égout par Eugène Belgrand à Paris.

Les économistes et les industriels ne sont pas non plus demeurés étrangers à cette nouvelle préoccupation.

Les premiers tentent d'affiner les instruments économiques de lutte contre la pollution tandis que les seconds proposent des matériels de plus en plus performants.

Dans le même temps, en rassemblant les compétences privées et publiques, ainsi qu'une plus large participation des citoyens, l'eau devient l'objet sinon d'un consensus du moins d'un projet collectif de grande ampleur.

Mais l'eau est aussi devenue un facteur limitant de notre économie.

Sans doute existe-t-il une réelle volonté politique d'apporter une réponse aux problèmes de l'eau au niveau national, dans les pays industrialisés notamment, mais l'enracinement dans la durée doit également se tocole de Montréal du 16 décembre 1987 sur la couche d’ozone, les menaces dues à l'augmentation des gaz à effet de serre dans l'atmosphère, les travaux de la Commission Brundtland qui a conduit à proposer le concept de " développement durable ", la déclaration économique du Sommet de l'Arche de juillet 1989, la conférence de Rio en 1992, la récente rencontre de Kyoto et certains ouvrages philosophiques, tels que le contrat naturel de Michel Serre, font prendre conscience à nos concitoyens et aux responsables politiques de l'état de santé, à maints égards inquiétant, de la planète.

En ce qui concerne l'eau, quelques étés secs avec interdiction d'arroser sa pelouse, de laver sa voiture, quelques pollutions accidentelles ou la mise en évidence de la présence en quantité anormale de tel ou tel élément toxique..., le tout relayé par des séries d'articles ou autres documents, ont fait passer la lutte contre la pollution de manifester au niveau international et notamment à l'égard des pays du Tiers Monde, tant les questions d'environnement et de développement sont intimement liées.

Or, les projets dans ce domaine requièrent d'importantes masses financières et ce, dans un délai souvent bref en raison de l'aggravation de la situation de ces pays, aggravation mise en évidence de manière quasiment emblématique par les phénomènes de sécheresse observés ces dernières années.

Et la question qui se pose alors est la suivante : les pays industrialisés sont-ils disposés - au-delà de déclaration de circonstance - à mettre à la disposition de la partie la plus pauvre (et la plus nombreuse) de la planète, une fraction de leurs ressources financières et de leur savoir-faire technologique ?

La réponse à cette question sera déterminante vis-à-vis de l'avenir. 